\title{
Current outcome of prioritized patients for kidney transplantation
}

\author{
Hideki Kanashiro, Fabio Cesar Miranda Torricelli, Renato Falci Junior, Affonso Celso Piovisan, Ioannis \\ Michel Antonopoulos, William Carlos Nahas
}

Renal Transplantation Unit, Division of Urology, Department of Surgery, Hospital das Clinicas, University of Sao Paulo Medical School, Sao Paulo, Brazil

\section{ABSTRACT}

Purpose: To analyze the outcome of deceased donor recipients given priority in allocation due to lack of access for dialysis and compare this data to the one obtained from non-prioritized deceased donor kidney transplant recipients.

Materials and Methods: we reviewed electronic charts of 31 patients submitted to kidney transplantation that were given priority in transplantation program due to lack of access for dialysis from January 2005 to December 2008. Immunological and surgical complications rates, and grafts and patients survival rates were analyzed. These data were compared to those obtained from 100 regular patients who underwent kidney transplantation without allocation priority during the same period.

Results: Overall surgical complication rate was $25.8 \%$ and $27 \%$ in the patients with priority in allocation and in the non-prioritized patients, respectively. There was no statistical significant difference for surgical complications ( $p=1.0$ ), immunological complications $(p=0.21)$ and graft survival $(p=0.19)$ rates between the groups. However, patient survival rate was statistically significant worse in prioritized patients ( $\mathrm{p}=0.05)$.

Conclusions: patients given priority in allocation owing to lack of access for dialysis have higher mortality rate when compared to those non-prioritized.

\section{ARTICLE INFO}

\section{Key words:}

Dialysis; health priorities; kidney; renal insufficiency; transplantation

Int Braz J Urol. 2012; 38: 389-94

Submitted for publication: November 04, 2011

\section{Accepted after revision:}

February 12, 2012

\section{INTRODUCTION}

Kidney transplantation is the gold standard treatment for end-stage renal disease (ESRD). However, the imbalance between organ supply and demand makes the implementation of equitable and effective organ allocation systems a major concern. In the United States, despite the increasing number of kidney transplants, more patients with ESRD are dying while waiting treatment (1). In developing countries, the situation is even worse, because only $6.2 \%$ of renal chronic patients are submitted to kidney transplantation (2).
Criteria for admission of patients to the waiting list, donor selection, tissue-typing methods, organ preservation and immunosuppressive protocols are the focus of intense debate in the literature (3-5). Efforts have been made to shorten the waiting time, to adjust for rare HLA phenotypes and homozygous and to guarantee an acceptable HLA match distribution in order to optimize the overall transplant success rate. Despite these efforts, kidney transplantation in select groups of patients may be performed with significant delay.

Priority in allocation of patients with ESRD is still a controversial subject. The best 
time for kidney transplantation of highly sensitized patients or for those who lack access for dialysis is still to be determined. Lack of access for dialysis is one of the criteria for priority in allocation to be given to patients for kidney transplantation. A patient is considered to be candidate for allocation priority when all but one access for dialysis had been unsuccessfully exploited. In our service, a patient is prioritized for transplantation when there is no more arteriovenous fistula and all veins (internal jugular vein, subclavian vein, and femoral vein) are no more available for catheter placement due to thrombosis. In summary, the patient is prioritized when he is in hemodialysis and the lost of his catheter will let him without access for dialysis. The aim of this study was to analyze the outcome of the kidney transplantation in patients put in priority in allocation owing to lack of access for dialysis and to compare complication rate as well as graft and patient survival rates to non-prioritized ones.

\section{MATERIALS AND METHODS}

We reviewed electronic charts of 31 prioritized patients for kidney transplantation due to the lack of access for dialysis from January 2005 to December 2008. These patients were compared to 100 non-prioritized ones, submitted to kidney transplantation during the same period. Only patients receiving their first graft from deceased donors were included in this analysis. Highly sensitized patients and patients with incomplete data or irregular follow-up were excluded. To compare similar groups, case-matched study was conducted, where study and control groups had the same time in dialysis (mean: 6.7 years) and similar donors and recipients mismatch. Basically, immunosuppression was achieved with the triple-drug regimen, based on tacrolimus, mycophenolate mofetil, and prednisone.

All grafts were positioned retroperitoneally through an extended inguinal incision. Vascular reconstruction was performed at the external iliac vessels. The urinary tract was reconstructed using the non-standardized Gregoir technique. Drains were not routinely used. Fascial closure was performed with running 0-0 polyglycolic acid sutures. Demographic data were analyzed with T-test. Com- plications rates were analyzed with Chi-square test or Fisher's Exact test. Both graft and patient survival rates were studied with the Kaplan-Meier survival curve and Logrank for survival comparison. Results were expressed in mean, standard deviation and range. Two-tailed values of $p<0.05$ were considered statistically significant. The mean followup was 37.7 (range 25 - 57) months, 36.0 months (range 25 - 57) in prioritized patients and 38.2 (range 27 - 52) months in non-prioritized ones.

\section{RESULTS}

Demographic data are summarized in $\mathrm{Ta}-$ ble-1. There was no statistically significant difference between the groups, regarding demographic data. Overall surgical complication rate was 25.8\% in the group of patients given priority in allocation. Eight surgical complications occurred in 7 $(22 \%)$ patients. Early and late surgical complications in prioritized patients were: three cases of vesicoureteral reflux with symptomatic urinary tract infection that were successfully treated with bulking agent injection, two cases of retroperitoneal hematoma that were surgically removed, one incisional hernia that was surgically repaired, one lymphocele that was surgically treated and one acute urinary retention that was treated with transurethral resection of the prostate. Eight (25\%) patients had humoral rejection and two (6.5\%) had the acute cellular one. Overall surgical complication rate in non-prioritized patients was 27\%. Twentyseven complications occurred in 24 patients (24\%). Early and late complications in the control group were: five vesicoureteral reflux with symptomatic urinary tract infection that were treated with bulking agent injection, one surgical site hematoma that was surgically drained, nine incisional hernias that were surgically repaired, three lymphoceles that were surgically treated, four urinary fistulas treated through ureteral reimplantation, one urethral stenosis that required internal urethrotomy and four renal artery stenosis that were successfully treated by angioplasty. Eighteen (18\%) patients had humoral rejection and two (2\%) patients had acute cellular one. The differences of surgical complication rate and graft rejection rate were not statistically significant between the groups as showed in Table-1. 
Table 1 - Demographic data and post-operative complications.

\begin{tabular}{|c|c|c|c|c|c|}
\hline & Prioritized & & Non-Prioritized & & $\mathrm{p}$ \\
\hline Number of Patients & 31 & $(23.6 \%)$ & 100 & $(76.4 \%)$ & - \\
\hline Gender (F) & 20 & $(64.5 \%)$ & 49 & $(49.0 \%)$ & 0.13 \\
\hline Age at Transplantation (years) & $42.1 \pm 15.7$ & $(10-67)$ & $47.0 \pm 14.6$ & $(6-75)$ & 0.13 \\
\hline Cold Ischemia Time & $18.1 \pm 10.9$ & $(10-44)$ & $17.1 \pm 13.4$ & $(10-36)$ & 0.18 \\
\hline Follow-up (months) & $36.0 \pm 8.0$ & $(25-57)$ & $38.2 \pm 7.2$ & $(27-52)$ & 0.24 \\
\hline Complications (n) & 18 & $(58.0 \%)$ & 47 & $(47.0 \%)$ & 0.28 \\
\hline Total Rejection & 10 & & 20 & & 0.21 \\
\hline Humoral rejection & 8 & $(25.8 \%)$ & 18 & $(18.0 \%)$ & 0.34 \\
\hline Celular rejection & 2 & $(6.5 \%)$ & 2 & $(2.0 \%)$ & 0.23 \\
\hline Total Surgical & 8 & $(25.8 \%)$ & 27 & $(27.0 \%)$ & 1.0 \\
\hline Vesicoureteral reflux & 3 & $(9.7 \%)$ & 5 & $(5.0 \%)$ & 0.39 \\
\hline Hematoma & 2 & $(6.5 \%)$ & 1 & $(1.0 \%)$ & 0.13 \\
\hline Incisional Hernia & 1 & $(3.2 \%)$ & 9 & $(9.0 \%)$ & 0.45 \\
\hline Voiding dysfunction & 1 & $(3.2 \%)$ & 0 & - & 0.23 \\
\hline Lymphocele & 1 & $(3.2 \%)$ & 3 & $(3.0 \%)$ & 1.0 \\
\hline Ureteral Leak / stenosis & 0 & - & 4 & $(4.0 \%)$ & 0.57 \\
\hline Urethral stenosis & 0 & - & 1 & $(1.0 \%)$ & 1.0 \\
\hline Renal artery stenosis & 0 & - & 4 & $(4.0 \%)$ & 0.57 \\
\hline
\end{tabular}

Seven (22\%) prioritized patients and fourteen (14\%) non-prioritized patients had graft loss, respectively. The causes of graft loss in prioritized and non-prioritized patients are synthesized in Table-2. There was no statistical significant difference for graft survival rate between the groups ( $p$ $=0.19$ ) - Figure-1. Regarding patients' outcome, eight (25.8\%) prioritized patients and 12 (12\%) non-prioritized patients died, respectively. The causes of death in prioritized patients were: six sepses, one uremia by lack of access for dialyses, and one bleeding. The causes of death in control group were: eleven sepses, and one pulmonary thromboembolism. The patient survival rate was 
Table 2 - Graft loss causes.

\begin{tabular}{lcc}
\hline & Prioritized & Non-Prioritized \\
\hline Total & 7 & 14 \\
Acute rejection & 3 & 5 \\
Renal tumor & 1 & 0 \\
Renal rupture & 1 & 1 \\
Venous thrombosis & 1 & 1 \\
Arterial thrombosis & 0 & 1 \\
Polyomavirus infection & 1 & 2 \\
Would infection with abscess & 0 & 2 \\
Bleeding & 0 & 1 \\
Glomerulosclerosis recurrence & 0 & 1 \\
\hline
\end{tabular}

Figure 1 - Graft survival.

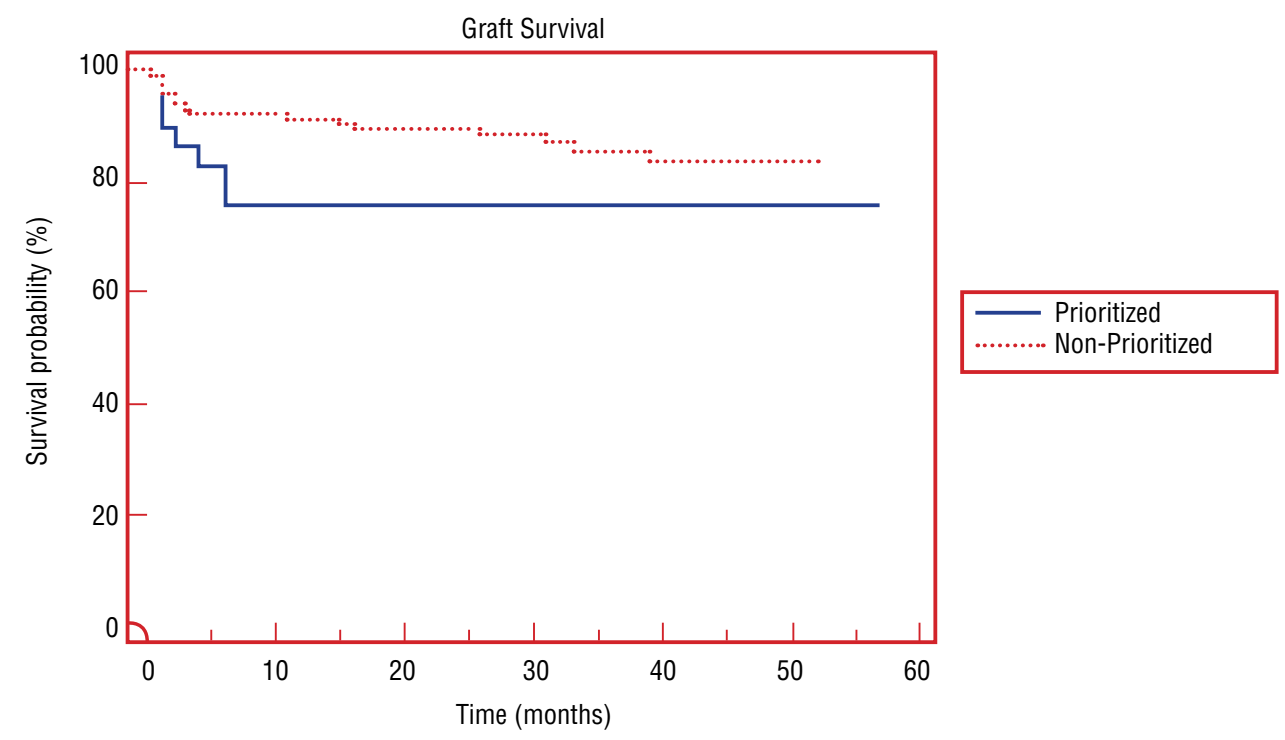

statistically significantly worse in prioritized patient $(\mathrm{p}=0.05)$ - Figure-2.

\section{DISCUSSION}

In our study, prioritized patients for kidney transplantation owing to absence of access for dialysis had worse outcome when compared to non-prioritized ones. Their graft survival rate in 5-years was similar, but prioritized ones had earlier graft dysfunction. Moreover, prioritized patients had significant lower survival rate. The worst outcome of prioritized patients is probably due to several factors, including clinical and surgical conditions at the time of kidney transplantation. Our data may not guarantee that earlier 
Figure 2 - Patient survival.

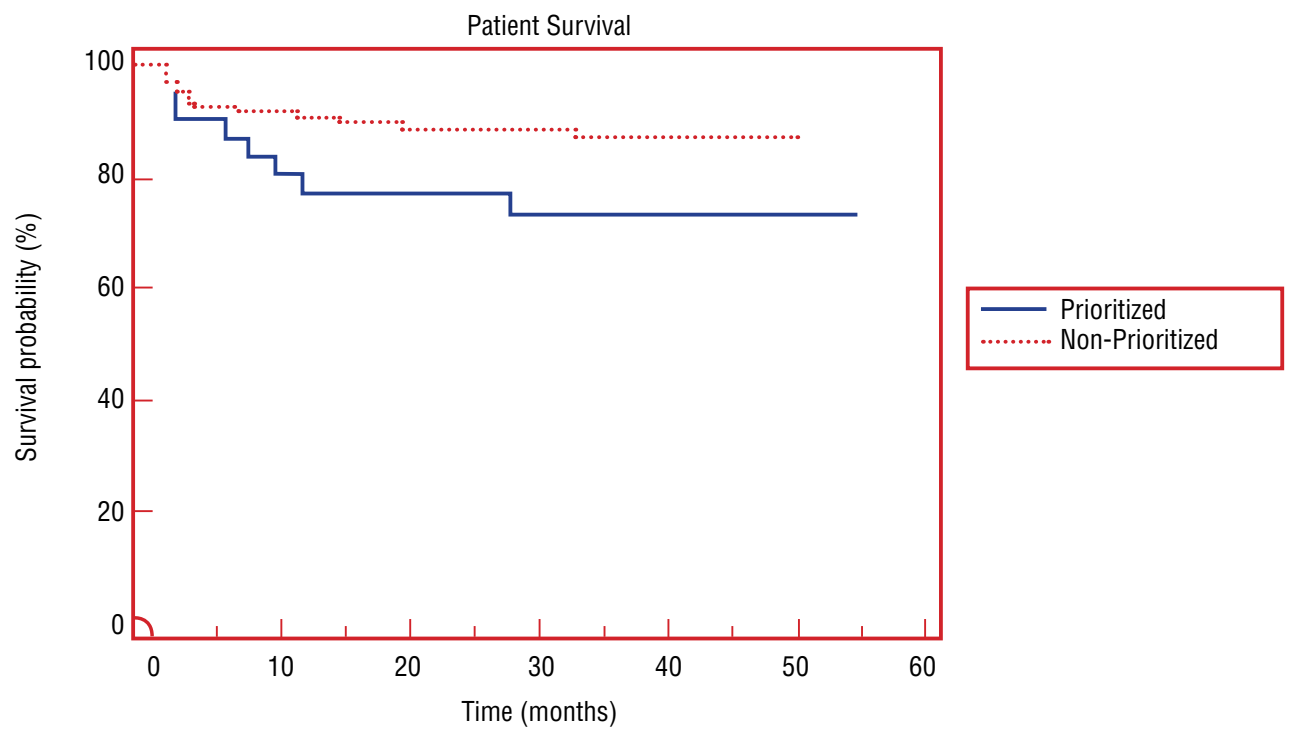

prioritization will put these patients in a better situation for transplantation, but it is an option. Perhaps prioritized patients may be benefited from earlier transplantation.

Great results achieved by kidney transplant programs have been motivating health care providers to search for new strategies to increase the number of deceased and living kidney donors and to find the best way to allocate these organs. In the United States, for single kidney transplants performed prior to 2008, patient survival rates at 5 years were $91 \%$ for recipients of living donors kidneys, 84\% for non-expanded criteria deceased donors, and 72\% for expanded criteria deceased donors (1). As a result of these good numbers, kidney transplantation centers have been increased around world; in the Latin America, kidney transplant rate increased from 3.7 per million population in 1987 to 15.4 per million population in 2006 (6).

Today, the rules to determine which candidate will receive an available organ are based on a score system that takes into account histocompatibility, blood group, age, clinical need, waiting time, negative crossmatch, and sensitization. Poli et al. (7) studied the factors impacting on deceased kidney graft survival and function, and after a multivariate analysis of a number of immunological, clinical, social, and administrative factors on transplant outcome concluded that younger donors, absence of pre-transplant transfusion, patient dialysis center and level of HLA match have a statistically significant positive association with excellent graft function at 4 years.

Dolan et al. (8) conducted an interesting study about how people wish to give priority based on certain characteristics of potential recipient of a donor kidney. Between the respondents, there was a clear consensus that one of the most important considerations is what will happen to the patient without the treatment, and so priority was given to those with poor prognosis. There was also a strong view that priority should be given to younger patients and to those with dependents. The time spent waiting for a transplant is also important, but less so. According to our results and taking into account the data published by Dolan et al., the patients with lack of access should be prioritized earlier, once the delay in kidney transplantation results in greater mortality rate. Maybe 
a better communication between nephrologists, urologists, and dialysis centers could put these patients in a better situation in the waiting list.

\section{CONCLUSION}

Patients given priority in allocation due to lack of access for dialysis have higher mortality rate when compared to those non-prioritized.

\section{CONFLICT OF INTEREST}

None declared.

\section{REFERENCES}

1. Axelrod DA, McCullough KP, Brewer ED, Becker BN, Segev DL, Rao PS: Kidney and pancreas transplantation in the United States, 1999-2008: the changing face of living donation. Am J Transplant. 2010; 10(4 Pt 2): 987-1002.

2. Cunha CB, León AC, Schramm JM, Carvalho MS, Souza Júnior PR, Chain R: Time to kidney transplantation in chronic renal failure patients in the State of Rio de Janeiro, Brazil, 1998-2002. Cad Saude Publica. 2007; 23: 805-13.

\section{EDITORIAL COMMENT}

The authors, who have ample experience with renal transplant, compared the data from a period of four years of 31 patients prioritized for transplant due to lack of access for dialysis and of 100 'regular' patients.

The retrospective study concluded that mortality in the group of prioritized patients was higher. The authors were unable to identify the cause of this difference, but it could have been caused by differences in the populations; not all of which were identified or analyzed.
3. Chang RW: How should cadaver kidneys be allocated? 1996; 17; 348: 453-4.

4. Guttmann RD: Cadaver kidneys: the rules of rationing. Lancet. 1996; 348: 456-7.

5. Starzl TE, Fung JJ: The politics of grafting cadaver kidneys. Lancet. 1996; 348: 454-5.

6. Cusumano AM, Gonzalez Bedat MC, García-García G, Maury Fernandez S, Lugon JR, Poblete Badal H, et al.: Latin American Dialysis and Renal Transplant Registry: 2008 Report (data 2006). Clin Nephrol. 2010; 74(S1): 3-8.

7. Poli F, Scalamogna M, Cardillo M, Porta E, Sirchia G: An algorithm for cadaver kidney allocation based on a multivariate analysis of factors impacting on cadaver kidney graft survival and function. Transpl Int. 2000; 13(Suppl 1): S259-62.

8. Dolan P, Shaw R: A note on a discussion group study of public preferences regarding priorities in the allocation of donor kidneys. Health Policy. 2004; 68: 31-6.

Correspondence address:

Dr. Fábio César Miranda Torricelli Av. Vereador Jose Diniz, 3300 / 208

Sao Paulo, SP, 04604-006, Brazil

Telephone: +55 11 5533-4900

E-mail: fabio_torri@yahoo.com.br

In any regard, the data points out the need for reviewing the criteria of patient prioritization for renal transplant, which is the merit of this study. Perhaps, as the authors pointed out, the patients could attain better outcomes if they received the transplant prior to the critical moment of the last access for dialysis. Alternatively, the prioritization criteria should be discarded as we are faced with this dire numerical picture of transplants in Brazil.

\author{
Dr. Lisias Nogueira Castilho \\ Instituto do Radium de Campinas \\ Campinas, SP, Brazil \\ E-mail: lisias@dglnet.com.br
}

